\title{
Low-Frequency Turbulence in a Linear Magnetized Plasma
}

\author{
B. N. Rogers ${ }^{1, *}$ and Paolo Ricci ${ }^{2, \dagger}$ \\ ${ }^{1}$ Department of Physics and Astronomy, Dartmouth College, Hanover, New Hampshire 03755, USA \\ ${ }^{2}$ Centre de Recherches en Physique des Plasmas-École Polytechnique Fédérale de Lausanne, \\ Association EURATOM-Confédération Suisse, CH-1015 Lausanne, Switzerland
}

(Received 23 March 2010; published 2 June 2010)

\begin{abstract}
Plasma turbulence in a linear device is explored for the first time with three-dimensional global twofluid simulations, focusing on the plasma parameters of the Large Plasma Device. Three instabilities are present in the simulations: the Kelvin-Helmholtz instability, a sheath-driven instability, and a resistive drift wave instability. The Kelvin-Helmholtz mode is shown to dominate the transport of plasma across the magnetic field. Simple scaling laws are obtained for the plasma profiles.
\end{abstract}

DOI: 10.1103/PhysRevLett.104.225002

PACS numbers: 52.35.Ra, 52.30.Ex, 52.35.Kt

Linear plasma devices (e.g., [1-6]) are of widespread interest to the plasma physics community because they allow the exploration of basic plasma phenomena without the complexities of magnetic curvature and shear. Of particular interest in this work, the Large Plasma Device (LAPD) experiment [1] creates a linear plasma approximately $18 \mathrm{~m}$ long and $30 \mathrm{~cm}$ in radius with straight magnetic field lines that terminate on the end walls. Among its many uses, this experiment has been applied to the study of turbulence and transport [7-11] due to modes such as the Kelvin-Helmholz (KH) instability and drift waves. These modes are of high interest because they are ubiquitous in magnetized plasmas, and drift waves, in particular, are believed to play a central role in the edge region of fusion devices. The latter topic is of great importance to the fusion community because edge turbulence largely governs the overall fusion performance of tokamaks and similar machines.

We present here global 3D two-fluid simulations of turbulence in a linear device with LAPD-like plasma parameters and Bohm sheath boundary conditions in the parallel direction. Spatially localized source terms are added to the density and temperature equations that mimic the top-hat-like shape of the source region in the LAPD experiments. Since the simulations evolve the full profiles of the various quantities with no separation made between "perturbations" and "equilibrium," they can explore the self-consistent evolution and structure of the plasma profiles in the presence of (1) the input of plasma and heat from the sources, (2) the cross-field transport produced by plasma instabilities (drift waves, for example), and (3) parallel losses at the sheaths where the magnetic field lines terminate on the end walls. Our simulation results are new and unexpected: we find that drift wave modes, although present, are not the main source of heat and particle crossfield transport in the device, nor are sheath-driven instabilities [12], which arise in the system from the sheath (Bohm) boundary conditions in the parallel direction [13]. Rather, the main agent of transport is the KH instability. This is a fully global mode, driven by shear in the equilib- rium electric potential arising from the sheath boundary conditions [13]: $e \phi \simeq \Lambda T_{e}$, where $\Lambda=\log \sqrt{m_{i} /\left(2 \pi m_{e}\right)} \simeq$ 3 . The nonlinear evolution of the KH mode produces largescale eddies that are the main source of cross-field profile relaxation.

For our study we use the electrostatic Braginskii equations [14] with $T_{i} \ll T_{e}$ and $\beta \ll 1$ :

$$
\begin{gathered}
\frac{d n}{d t}=-\frac{\partial\left(n V_{\| e}\right)}{\partial z}+S_{n}, \quad \frac{d V_{\| i}}{d t}=-V_{\| i} \frac{\partial V_{\| i}}{\partial z}-\frac{1}{n} \frac{\partial p_{e}}{\partial z}, \\
\frac{d \nabla_{\perp}^{2} \phi}{d t}=-V_{\| i} \frac{\partial \nabla_{\perp}^{2} \phi}{\partial z}+\frac{m_{i} \Omega_{c i}^{2}}{e^{2} n} \frac{\partial j_{\|}}{\partial z}, \\
\frac{d T_{e}}{d t}=\frac{2}{3} \frac{T_{e}}{e n} 0.71 \frac{\partial j_{\|}}{\partial z}-\frac{2}{3} T_{e} \frac{\partial V_{\| e}}{\partial z}-V_{\| e} \frac{\partial T_{e}}{\partial z}+S_{T}, \\
m_{e} \frac{d V_{\| e}}{d t}=-m_{e} V_{\| e} \frac{\partial V_{\| e}}{\partial z}-\frac{T_{e}}{n} \frac{\partial n}{\partial z}+e \frac{\partial \phi}{\partial z}-1.71 \frac{\partial T_{e}}{\partial z}+\frac{e j_{\|}}{\sigma_{\|}},
\end{gathered}
$$

where $p_{e}=n T_{e},[a, b]=\partial_{x} a \partial_{y} b-\partial_{y} a \partial_{x} b, d f / d t=$ $\partial f / \partial t-(c / B)[\phi, f], \quad j_{\|}=e n\left(V_{\| i}-V_{\| e}\right), \quad \Omega_{c i}=$ $e B /\left(m_{i} c\right)$. The $z$ is the coordinate parallel to $B$. We solve Eqs. (1)-(4) on a field-aligned grid using a finite difference scheme with Runge-Kutta time stepping and small numerical diffusion terms. The computational domain has a rectangular shape spanning $(-L / 2, L / 2), L=100 \rho_{s 0}$ in the perpendicular directions and $\left(-L_{z} / 2, L_{z} / 2\right)$ in the parallel direction with $n_{x}=n_{y}=1024, n_{z}=64$. We use profiles for the density and temperature sources $S_{n}, S_{T}$ that are similar to the top-hat-like source profiles in LAPD: $S_{n, T}=$ $S_{0 n, T}\left\{1-\tanh \left[\left(r-r_{s}\right) / L_{s}\right]\right\} / 2$ where $r=\sqrt{x^{2}+y^{2}}$. We consider the nominal values for a helium LAPD plasma: $L_{z} \simeq 18 \mathrm{~m}, \Omega_{c i} \sim 960 \mathrm{kHz}, R \simeq 0.5 \mathrm{~m}$ (the approximate radius of the LAPD plasma chamber), $r_{s} \simeq 28 \mathrm{~cm}, n_{0} \sim$ $2 \times 10^{12} \mathrm{~cm}^{-3}, \quad T_{e 0} \sim 6 \mathrm{eV}, \quad c_{s 0}=\sqrt{T_{e 0} / m_{i}} \sim 1.2 \times$ $10^{6} \mathrm{~cm} / \mathrm{s}, \quad \rho_{s 0}=c_{s 0} / \Omega_{c i} \sim 1.4 \mathrm{~cm}, \quad c_{s 0} / R \sim 2.4 \mathrm{MHz}$. The parameters used in the simulations are $S_{0 n}=$ $0.03 n_{0} c_{s 0} / R, \quad S_{0 T}=0.03 T_{e 0} c_{s 0} / R, \quad L_{s}=0.5 \rho_{s 0}$, 


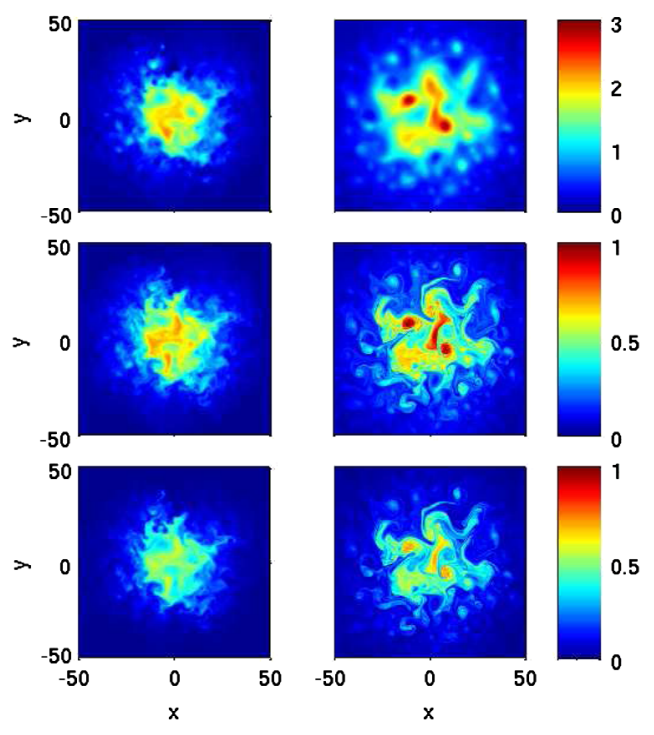

FIG. 1 (color online). Plots of $\phi$ (top), $T_{e}$ (center), and $n$ (bottom) perpendicular to $B$ in 3D (left) and 2D (right) simulations.

$r_{s}=20 \rho_{s 0}, \quad m_{i} / m_{e}=400, \quad \Lambda=3, \quad R / \rho_{s 0}=40, \quad \nu=$ $e^{2} n_{0} R /\left(m_{i} c_{s 0} \sigma_{\|}\right)=0.03, L_{z}=36 R$. In the figures we normalize $n$ to $n_{0}, T_{e}$ to $T_{e 0}, \phi$ to $T_{e 0} / e$, perpendicular lengths to $\rho_{s 0}$, parallel lengths to $R$, and time to $R / c_{s 0}$. In the perpendicular directions, we make the computational domain large enough $\left(L=100 \rho_{s 0} \sim 1.4 \mathrm{~m}\right)$ so that essentially no plasma reaches the walls and thus the transverse boundary conditions have no impact on the simulations. This simplifies the simulations but is different from the experiments, which have a somewhat smaller circular cross section $(\sim 1$ m diameter). Another simplification concerns the sources: in the simulations the sources are uniform in the parallel direction, and standard Bohm boundary conditions $V_{\| i}= \pm c_{s}, \quad V_{\| e}= \pm c_{s} \exp \left(\Lambda-e \phi / T_{e}\right)$ are applied at the end walls $z= \pm L_{z} / 2$. In the LAPD experiments, however, the situation is more complicated. Energetic electrons are injected by an anode-cathode arrangement at one end [1], and the potential of the anode and cathode can be biased relative to the walls of the vacuum chamber. Data can be taken during the active period of the source or in the afterglow phase, in which the temperature falls rapidly as the plasma is lost in the parallel direction. More research is needed to determine how the boundary conditions of the simulations and operating configuration of the experiments can best be matched. For this reason, our results should be regarded as only the first step toward modeling turbulence in the LAPD.

Figure 1 (left-hand panels) shows typical cuts of $\phi, T_{e}$, and $n$ perpendicular to $B$ through the center $(z=0)$ of a $3 \mathrm{D}$ simulation. The corresponding long-time averages, also at $z=0$, are shown in Fig. 2 (solid lines). The time averages of $\phi$ and $T_{e}$ satisfy $e \phi \simeq \Lambda T_{e}$ as noted earlier. The density and temperature equations are sufficiently similar so that the normalized density and temperature profiles are nearly the same: $T_{e} \propto n, \tilde{T}_{e} / T_{e} \sim \tilde{n} / n$. Aside from the fluctua-
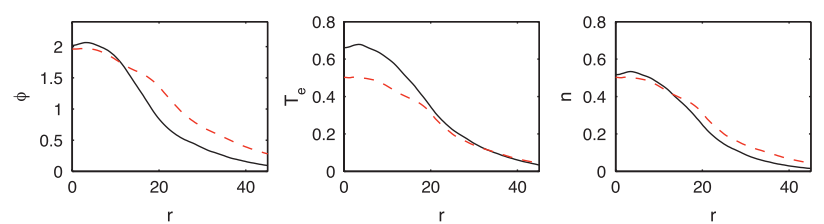

FIG. 2 (color online). Cuts of the long-time averages of $\phi$ (left), $T_{e}$ (center), and $n$ (right) in 3D (black solid lines) and 2D (red dashed lines) simulations.

tions, the profiles of $\phi, T_{e}$ and $n$ are approximately constant in the parallel direction, as can be seen from the parallel slices through $y=0$ plotted in Fig. 3 .

The turbulent fluctuations in these figures stem from three main instabilities. The largest perpendicular structures in Fig. 1 correspond to the most unstable KH modes in the system. These are global modes with a radial extent comparable to $L_{0}$, the radial gradient scale length of $\phi$, poloidal wave numbers $k_{\theta} L_{0} \sim 1$, and $k_{\|} \simeq 0$. The shorter scale activity in the plots is produced by drift waves and, of lesser importance at these parameters, sheath modes. In contrast to the $\mathrm{KH}$ and sheath modes, drift waves require finite $k_{\|}$to be unstable. The peak drift wave linear growth rate in our system is $\gamma \simeq 0.085(1+1.71 \eta) c_{s} / L_{n}, \quad \eta=$ $L_{n} / L_{T}$, for $k_{\|} \simeq 0.24 \sqrt{\nu /\left(c_{s} L_{p}\right)}$ and $k_{y} \rho_{s} \simeq 0.57$. The most unstable parallel wavelengths for typical LAPD parameters are comparable to the length of the machine, $k_{\|} \sim 2 \pi / L_{z}$.

The instantaneous profiles of the density, temperature, and electric potential are typically steeper, by about a factor of 2, than the smooth, Gaussian-like time-averaged profiles shown in Fig. 2. The difference between the two is caused by the global nature of the KH fluctuations, which are continually excited in the steep gradient region and cause an order-unity flattening of the local gradients. Figure 4 (left-hand panel) shows a plot of $L_{0}$, where $1 / L_{0}=\max \left(d_{r} \phi / \phi\right)$, taken from a cut along $y=0$ of the instantaneous $\phi$ profile (solid lower line, $L_{0} \sim 3 \rho_{s 0}$ ) compared to that computed from the time-averaged profile

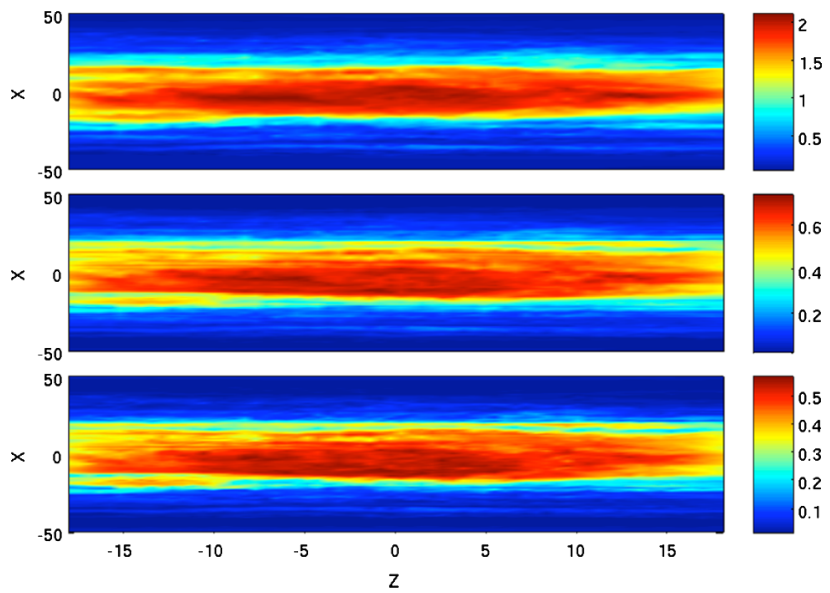

FIG. 3 (color online). Plots of $\phi$ (top), $T_{e}$ (center), and $n$ (bottom) parallel to $B$ in $3 \mathrm{D}$ simulations. 

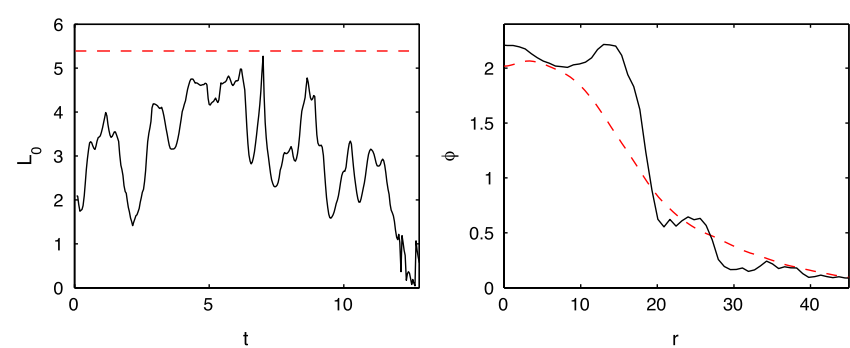

FIG. 4 (color online). Left-hand panel: The steepest $\phi$ profile scale length obtained from a cut of the instantaneous $\phi$ profiles (lower solid line) and the same obtained from the time-averaged profile (upper dashed line). Right-hand panel: Long-time average (dashed line) and instantaneous (solid line) $\phi$ profile.

(upper dashed line, $L_{0} \sim 5.5 \rho_{s 0}$ ). As shown in Fig. 4 (right-hand panel), in contrast to the rather broad timeaveraged profiles, the instantaneous profiles retain an approximate top-hat-like or hyperbolic-tangent shape of the form $\phi=\phi_{\max }\left\{1-\tanh \left[\left(r-r_{s}\right) / L_{0}\right]\right\} / 2$ with $L_{0} \sim 3 \rho_{s 0}$ and a radial extent similar to that of the sources, $r \sim r_{s}$. Similar expressions apply to $n$ and $T_{e}$ with $L_{n} \sim L_{T} \sim L_{0}$. Tests show $L_{0}$ is not dependent on the much steeper source profiles $\left(L_{s}=0.5 \rho_{s 0}\right)$ but is rather determined by the strength of the cross-field transport (discussed below).

Consistent with the simulations, the numerical linear stability analysis of such hyperbolic-tangent profiles predicts a band of unstable $\mathrm{KH}$ modes centered on $k_{\theta} L_{0} \simeq 1$, or poloidal mode number $m=r_{s} / L_{0} \sim 7$. This prediction is also consistent with a simple slab $\mathrm{KH}$ analysis of such profiles (the Bickley jet) [15], albeit with growth rates that are reduced by sheath effects below the maximum slab values $\gamma \simeq 0.1(c / B) \phi_{\max } / L_{0}^{2}$. In contrast to this good agreement, the full numerical stability analysis of the more gradual, Gaussian-like time-averaged profiles shown in Fig. 2 indicates they are linearly stable to all $k_{\|}=0$ modes: tests show the KH mode is stabilized by sheath effects, while the sheath-driven mode is stabilized by velocity shear. This is in contradiction to the presence of the unstable $k_{\|} \simeq 0$ fluctuations in the simulations and underscores that these fluctuations are generated by the steeper instantaneous profiles rather than the long-time averages.

Turning to the issue of turbulent transport, our study indicates that the $\mathrm{KH}$ modes, rather than drift waves or sheath modes, provide the dominant cross-field transport channel in the simulations. This conclusion stems from several tests. First, when the KH drive is eliminated from the simulations, the profiles dramatically steepen; see Fig. 5, right-hand panel. The KH drive arises from the convective term $\propto\left[\phi, \nabla_{\perp}^{2} \phi\right]$ on the left-hand side of vorticity Eq. (2), and has been eliminated in the simulation by the replacement $\left[\phi, \nabla_{\perp}^{2} \phi\right] \rightarrow\left[\langle\phi\rangle_{\theta}, \nabla_{\perp}^{2} \phi\right]$, where $\langle\phi\rangle_{\theta}$ denotes an average over the polar angle $\theta$. Similar results are obtained if the $\left[\phi, \nabla_{\perp}^{2} \phi\right]$ term is completely dropped from the simulation. The extreme steepening of the profiles following the suppression of the $\mathrm{KH}$ mode suggests that the cross-field transport arising from drift wave and sheath modes is quite weak.

Another way to assess the importance of the $k_{\|} \neq 0$ drift wave modes is to eliminate them from the simulations while preserving the $k_{\|}=0 \mathrm{KH}$ and sheath modes. This can be done by integrating the 3D equations along the parallel direction to obtain a 2D system in which only the $k_{\|}=0$ structures survive [16]:

$$
\begin{gathered}
\frac{d n}{d t}=-\sigma \frac{n c_{s}}{R} \exp \left(\Lambda-e \phi / T_{e}\right)+S_{n}, \quad \sigma=1.5 R / L_{z} \\
\frac{d \nabla^{2} \phi}{d t}=\sigma \frac{c_{s} m_{i} \Omega_{i}^{2}}{e R}\left[1-\exp \left(\Lambda-e \phi / T_{e}\right)\right]
\end{gathered}
$$

$$
\frac{d T_{e}}{d t}=-\sigma \frac{2}{3} \frac{T_{e} c_{s}}{R}\left[1.71 \exp \left(\Lambda-e \phi / T_{e}\right)-0.71\right]+S_{T}
$$

This $2 \mathrm{D}$ reduction is only approximate: in the 3D simulations, $\phi, T_{e}$, and $n$ drop by about $20 \%$ in the parallel direction from the midplane to the sheath edge, and to obtain a 2D model it is necessary to neglect such variations as well as various other small terms. Nevertheless, semiquantitative agreement between the two models is obtained: Fig. 1 (lower panels) shows typical plots of $\phi$, $T_{e}$, and $n$ in the 2D model. The differences between the fluctuations in the 2D and 3D plots arise mainly from the presence of drift waves in the latter, which lead to a diffusive spreading of the $\mathrm{KH}$ vortices. The drift wave modes, however, do not substantially relax either the long-time average profiles [see Fig. 2, dashed lines, for the $2 \mathrm{D}$ case] or the steeper peak gradients of the instantaneous profiles (not shown). The similarity of the 2D and 3D profiles provides further evidence that the transport associated with drift waves in 3D is secondary.

It is possible obtain simple scaling laws for the peak values of the profiles $\phi_{\max }, T_{e, \max }, n_{\max }$, as well as the gradient scale length $L_{0}$. Since $L_{0}<r_{s}$, the height of the $T_{e}$ and $n$ profiles may be estimated by balancing the source terms $S_{T}$ and $S_{n}$ with the parallel loss terms (proportional to $1 / L_{z}$ ). To a first approximation (valid to roughly the $30 \%$ level), the exponential factors in the latter may be replaced by unity given $e \phi \sim \Lambda T_{e}$. In the case of $T_{e}$ this balance yields $2 \sigma T_{e} c_{s} /(3 R) \sim S_{T}$ or $T_{e, \max } \sim$ $m_{i}^{1 / 3}\left[3 R S_{T} /(2 \sigma)\right]^{2 / 3}$. This estimate, denoted by $T_{e, a n}$, is plotted versus the measured values in the simulations in Fig. 6(a), showing reasonably good agreement $\left(T_{e, \max } \simeq\right.$ $\left.0.6 T_{e, a n}\right)$. Given $T_{e, \max }$, the peak value $\phi$ is determined by $e \phi_{\max } \simeq \Lambda T_{e, \max }$. A similar result holds for $n_{\max }$.

Beyond the source region the profiles decay in the radial direction over the scale length $L_{0}$, which is determined by the strength of the cross-field transport due to the $\mathrm{KH}$ modes. As noted earlier, these global modes have radial envelopes comparable to $L_{0}$ and $k_{\theta} L_{0} \sim 1$ [see Fig. 6(b)] and in the nonlinear stage produce large vortices that 

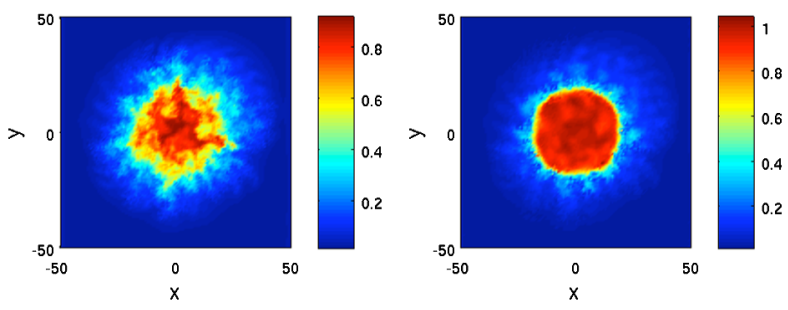

FIG. 5 (color online). The $T_{e}$ profile before (left) and after (right) the $\mathrm{KH}$ drive is turned off in a $3 \mathrm{D}$ simulation.

effectively mix the plasma inside and outside the steep gradient region. As shown in Fig. 6(c) this large-scale mixing produces order-unity rms temperature, density, and potential fluctuations in the steep gradient region: $\tilde{T}_{e} \sim c_{0} T_{e, \max }, \quad \tilde{n} \sim c_{0} n_{\max }, \quad \tilde{\phi} \sim c_{0} \phi_{\max } \sim c_{0} \Lambda T_{e, \max }$, where $c_{0} \sim 0.3$. The $\phi$ fluctuations are approximately consistent not only with $e \phi \sim \Lambda T_{e}$, but are also what one would expect from the nonlinear $\mathrm{KH}$ instability: balancing the two main terms in the vorticity equation $\partial_{t} \nabla_{\perp}^{2} \phi \sim(c / B)\left[\phi, \nabla_{\perp}^{2} \phi\right]$ with $\partial_{x} \sim \partial_{y} \sim 1 / L_{0}$ yields $\gamma \sim(c / B) \tilde{\phi} / L_{0}^{2}$. With the slab KH mode growth rate discussed earlier, $\gamma \sim 0.1(c / B) \phi_{\max } / L_{0}^{2}$, this reduces to $\tilde{\phi} \sim$ $0.1 \phi_{\max }$.

Given the strength of the KH fluctuations, the profile scale length $L_{0}$ may be estimated from the radial transport equation for $T_{e}$. Averaging Eq. (7) over $t$ and $\theta$, one obtains

$$
\frac{1}{r} \frac{\partial\left(r \Gamma_{T}\right)}{\partial r}=-\frac{2 \sigma T_{e} c_{s}}{3 R}+S_{T}, \quad \Gamma_{T}=\left\langle\tilde{T}_{e} \tilde{V}_{r}\right\rangle_{\theta, t} .
$$

Just outside the source region where $S_{T}=0$ and $\partial_{r} \Gamma_{T} \sim$ $-\Gamma_{T} / L_{0}$, this yields $\Gamma_{T} / L_{0} \sim 2 \sigma T_{e} c_{s} /(3 R)$, or estimating $\Gamma_{T} \sim k_{\theta}(c / B) \tilde{\phi} \tilde{T}_{e} \sim c_{0}^{2} \rho_{s} \Lambda c_{s} T_{e, \max } / L_{0}: \quad L_{0}=$ $0.37 \sqrt{\Lambda \rho_{s} R / \sigma}$. Figure 6(d) shows a plot of this result (normalized by $\rho_{s 0}$ and denoted $L_{0, \text { an }}$ ) versus the measured values, demonstrating good agreement.

Surprisingly, the parametric dependence of $\Gamma_{T}$ due to $\mathrm{KH}$ modes is the same as what one would expect for drift waves. Estimating $\gamma \tilde{T}_{e} \sim \tilde{V}_{r} T_{e, \max } / L_{0}$ and [17] $\partial_{r} \tilde{T}_{e} \sim$ $k_{r} \tilde{T}_{e} \sim T_{e, \max } / L_{0}$ with $k_{r} \sim \sqrt{k_{\theta} / L_{0}}, \quad k_{\theta} \sim 1 / \rho_{s}, \quad \gamma \sim$ $0.1 c_{s} / L_{0}$, one obtains $\Gamma_{T} \sim \tilde{V}_{r} \tilde{T} \sim 0.1 c_{s} \rho_{s} T_{e, \max } / L_{0}$ as above. It is therefore not clear whether there is any parameter regime in which drift wave transport can dominate the plasma.

Most of the data relating to the KH instability in LAPD has been obtained by externally biasing the plasma relative to the chamber wall $[10,11]$ —an effect not included in this work. When this biasing is weak or absent, in agreement with the simulations presented here, the scale lengths of the various profiles become comparable to each other. It has been speculated [11] that the transport in this weak shear case is due to either a combination of $\mathrm{KH}$ and drift wave modes or to drift waves alone. Our findings, on the other hand, suggest that the drift wave component of the transport is secondary. Strong KH mode activity has also been
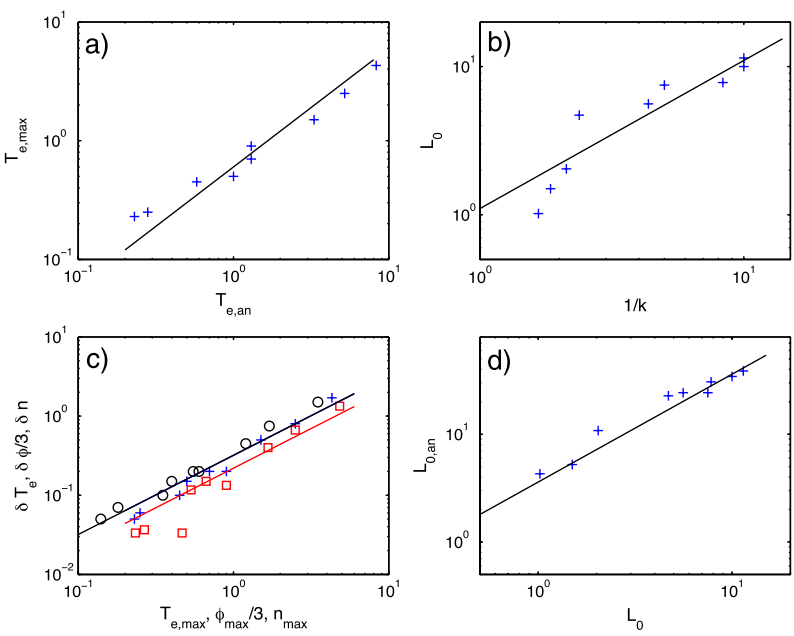

FIG. 6 (color online). (a) $T_{e, \max }$ vs $T_{e, a n}$, the line denotes $T_{e, \max }=0.6 T_{e, a n}$. (b) $L_{0}$ vs measured values of $1 / k_{\theta}$, the line denotes $L_{0}=1.1 / k_{\theta}$. (c) $\tilde{T}_{e}$ vs $T_{e, \max }$ (crosses), $\tilde{n}$ vs $n_{\max }$ (circles), $\tilde{\phi} / 3$ vs $\phi_{\max } / 3$ (squares), the black line denotes $\tilde{T}_{e}=$ $0.32 T_{e, \max }$ and $\tilde{n}=0.32 n_{\max }$, the red line denotes $\tilde{\phi}=$ $0.22 \phi_{\max }$. (d) $L_{0, \text { an }}$ vs simulation values, the line denotes $L_{0, \text { an }}=$ $3.6 L_{0}$.

identified in the Mirabelle experiment [3] when it is run with a top-hat-like source profile. As noted earlier, however, the parallel boundary conditions and source mechanisms in the simulations are much simpler than those in the experiments, and more work is needed to carry out detailed comparisons to the experiments.

This work was supported by grants from DoE/ESPCoR/ CICART, and by the "Fonds National Suisse de la Recherche Scientifique."

*barrett.rogers@dartmouth.edu

paolo.ricci@epfl.ch

[1] W. Gekelman et al., Rev. Sci. Instrum. 62, 2875 (1991).

[2] G. Tynan et al., Phys. Plasmas 11, 5195 (2004).

[3] F. Brochard et al., Phys. Plasmas 12, 062104 (2005).

[4] G. A. Navratil et al., Phys. Rev. Lett. 47, 1057 (1981).

[5] E. Wallace et al., Rev. Sci. Instrum. 75, 5160 (2004).

[6] T. Yamada et al., Nature Phys. 4, 721 (2008).

[7] T. Carter, Phys. Plasmas 13, 010701 (2006).

[8] D. Pace et al., Phys. Plasmas 15, 122304 (2008).

[9] T. Carter and J. Maggs, Phys. Plasmas 16, 012304 (2009).

[10] W. Horton et al., Phys. Plasmas 12, 022303 (2005).

[11] J. Perez et al., Phys. Plasmas 13, 055701 (2006).

[12] H. L. Berk et al., Nucl. Fusion 33, 263 (1993).

[13] P. Stangeby, The Plasma Boundary of Magnetic Fusion Devices (Institute of Physics, Philadelphia, PA, 2000).

[14] P. Ricci and B. Rogers, Phys. Plasmas 16, 092307 (2009).

[15] D. Biskamp, Magnetic Reconnection in Plasmas (Cambridge University Press, Cambridge, England, 2000).

[16] P. Ricci et al., Phys. Rev. Lett. 100, 225002 (2008).

[17] B. Rogers and W. Dorland, Phys. Plasmas 12, 062511 (2005). 